\title{
The longitudinal relation between brain lesion load and atrophy in multiple sclerosis: a 14 year follow up study
}

\author{
D T Chard, P A Brex, O Ciccarelli, C M Griffin, G J M Parker, C Dalton, D R Altmann, A J Thompson, \\ D H Miller
}

See end of article for authors' affiliations

Correspondence to:

Correspondence to:
Professor D H Miller, NMR Research Unit, Institute of Neurology, Queen Square, London WCIN 3BG UK:

d.miller@ion.ucl.ac.uk

Received

22 November 2002

In revised form

17 April 2003

Accepted 25 April 2003

Background: Studies have suggested that T2 lesion activity is prominent in early relapsing-remitting multiple sclerosis, whereas brain atrophy, while seen early, appears more evident in later progressive disease. The temporal relation between these processes remains unclear.

Objective: To explore the association between changing brain lesion loads and subsequent tissue atrophy in multiple sclerosis.

Methods: 28 subjects with clinically probable or definite multiple sclerosis (mean age 46.0 years; 17 female and 11 male) were followed for 14 years after first onset of symptoms. T2 lesion loads were estimated soon after symptom onset and at around five, 10, and 14 years later. Disease related atrophy was estimated at the 14 year follow up by comparing brain tissue volumes (proportional to total intracranial volumes) determined in the multiple sclerosis group with data from 29 normal control subjects (mean age 36.7 years; 16 female, 13 male) using multiple linear regression analyses to allow for differences in age and sex distributions.

Results: Change in lesion load in the first five years was more closely correlated to disease related brain atrophy at 14 years than later changes in lesion load, although the correlation was only moderate (Spearman correlation $=-0.528, \mathrm{p}=0.004$ ).

Conclusions: From this, it appears that early rather than later focal lesion accumulation relates to subsequent brain atrophy, but factors unconnected directly with lesion formation probably also play a significant role in determining such atrophy.

S tudies have shown that brain atrophy occurs in multiple sclerosis in excess of that observed in unaffected subjects, and this may be related to brain lesion loads. However, the temporal relation between these processes remains unclear. Does focal lesion genesis lead immediately to, or initiate a process of, subsequent brain atrophy? Does the stage of the disease during which it occurs influence atrophy? What is the strength of the relation between lesions and atrophy after long term follow up? Answering such questions will help our understanding of the pathological substrates of disability and clarify the targets and timing of therapeutic interventions

To address aspects of these questions, we looked at the relation between fractional brain tissue volumes, estimated a mean of 14 years after the onset of a clinically isolated syndrome, and lesion loads measured soon after onset and after 5,10 , and 14 years. This would provide insight into the temporal relation between lesion genesis and atrophy over a uniquely long period.

\section{METHODS \\ Subjects}

Data came from 29 normal control subjects ( 16 female and 13 male; mean age 36.7 years (range, 23.2 to 55.2 years)) and 28 subjects followed up for a mean of 13.8 years (range 12.4 to 15.4 years) after developing a clinically isolated syndrome ( 17 female, 11 male; mean age at 14 year follow up scan, 46.0 years (range, 35.3 to 62.9 years); median expanded disability status scale (EDSS) score, 2.0 (range 0.0 to 7.5 )). At the 14 year follow up, 20 had relapsing-remitting, three had secondary progressive, and five had clinically probable multiple sclerosis, as defined using the Poser criteria. ${ }^{3}$
The subjects with multiple sclerosis were derived from an initial cohort of 109 individuals who were recruited and followed up for one year at the National Hospital at Queen Square or Moorfields Eye Hospital between 1984 and 1987.. ${ }^{5}$ The original inclusion criteria were:

- a clinically isolated syndrome defined as an acute or subacute episode suggestive of demyelination, affecting the optic nerves, brain stem, or spinal cord;

- age at presentation between 10 and 50 years (to avoid normal age related changes on magnetic resonance imaging (MRI));

- no alternative diagnosis indicated by appropriate investigations.

Eighty nine of these subjects were followed up to a mean of 5.3 years, ${ }^{67}$ and 81 to a mean of 9.7 years. ${ }^{8}$ The present cohort of 28 represents a subset of the 71 ( of whom 53 had clinically definite or probable multiple sclerosis) followed up to a mean of 14.1 years, ${ }^{9}$ who had not been treated with $\beta$ interferon, had complete MRI T2 lesion load data from all time points, and had volumetric brain imaging at 14 years yielding usable tissue segmentations.

Of the 53 with clinically definite or probable multiple sclerosis, three died before the 14 year follow up and three had been treated with $\beta$ interferon, leaving an available untreated cohort of 47 subjects (median EDSS 2.5, range 0.0 to 9.5; mean disease duration 14.1 years, range 12.5 to 16.8 ). $\mathrm{T} 2$ lesion load data at 14 years were available for 40 of these subjects (median T2 lesion load, $11.1 \mathrm{ml}$, mean $14.7 \mathrm{ml}$, range 0.5 to $52.6 \mathrm{ml}$ ). Twenty nine of these subjects also had lesion load data available from the baseline examination and from the five and 10 year follow up, along with volumetric imaging data at 14 years. Tissue segmentation was assessed 
as being inadequate in one of these subjects (T2 lesion load at the 14 year follow up, $3.2 \mathrm{ml}$ ).

\section{Scan acquisition}

Subjects were scanned at baseline and at five years using a Picker 0.5 Tesla system (Picker Inc, Cleveland, Ohio, USA), and at 10 and 14 years using a General Electric Signa 1.5 Tesla system (General Electric Inc, Milwaukee, Wisconsin, USA). T2 weighted structural images were acquired at all time points. At baseline and five years, spin echo images were acquired (repetition time (TR), $2000 \mathrm{~ms}$; echo time (TE), 60 $\mathrm{ms})$. The slice thickness for these scans was either 5 or $10 \mathrm{~mm}$ at baseline and consistently $5 \mathrm{~mm}$ at five years. At 10 years, dual echo spin echo images were acquired (TR 2000 $\mathrm{ms}$, TE 30/90 ms, slice thickness $5 \mathrm{~mm}$ ).

At the 14 year follow up scan, a three dimensional inversion prepared fast spoiled gradient recall (3D-FSPGR) sequence was acquired (TR $16 \mathrm{~ms}$, TE $4.2 \mathrm{~ms}$, inversion time $450 \mathrm{~ms}$, matrix $160 \times 256$ interpolated to a matrix of $192 \times 256$, field of view $225 \times 300 \mathrm{~mm}$, final in plane resolution $1.2 \mathrm{~mm}$ by $1.2 \mathrm{~mm}$, with $124 \quad 1.5 \mathrm{~mm}$ slices covering the whole brain), along with a dual echo fast spin echo (FSE) sequence (TR $2000 \mathrm{~ms}$, TE 19/95 ms, slice thickness $5 \mathrm{~mm}$ ).

\section{Image processing}

\section{Lesion load determination}

T2 lesion loads (whole brain lesion volumes) were determined using a previously described method ${ }^{10}$ based on tools within Dispimage. ${ }^{11}$

\section{Whole brain and tissue specific fractional volume determination}

Using a previously described method based on tissue segmentations derived from SPM99 (Wellcome Department of Cognitive Neurology, Institute of Neurology, London, UK) and semiautomatic lesion segmentations generated using tools within Dispimage, ${ }^{11}$ grey matter, normal appearing white matter, cerebrospinal fluid (CSF), and lesion volumes were estimated from the 3D-FSPGR images. ${ }^{12}$ Segmentations were inspected to confirm adequate extraction of the brain tissues and CSF. Results were assessed as fractions of total intracranial volume, determined by adding the grey matter, normal appearing white matter, lesion, and CSF volumes. Brain parenchymal fraction (BPF) was calculated as grey matter plus normal appearing white matter plus lesion volumes, all divided by total intracranial volume.

\section{Statistical analyses}

Statistical analyses were done using SPSS 10.0 (SPSS Inc, Chicago, Illinois, USA) and Stata 7.0 (Stata Corporation, College Station, Texas, USA). Age and sex effects on tissue volumes were allowed for by calculating differences between predicted (for a given subject's age and sex) and measured $\mathrm{BPF}$, yielding estimates of disease related atrophy. Predicted normal values were estimated using multiple (linear) regression models which included sex as a categorical variable, age as a continuous covariate, and an age-sex interaction term applied to the control data.

Fractional tissue volumes from subjects with clinically definite or probable multiple sclerosis were compared with control subjects using multiple regression models that included age as a continuous covariate, sex, and disease status (multiple sclerosis or control) as categorical variables, and an age-sex interaction term.

Spearman correlations were estimated, along with confidence intervals ${ }^{13}$ between lesion load and tissue volume parameters. Multiple regression models were used to estimate the relative contribution of changing T2 lesion loads between the time points studied and estimated atrophy at the
Table 1 Lesion loads in the 28 subjects followed up over 14 years

\begin{tabular}{ll}
\hline Time point or interval & T2 lesion load $(\mathrm{ml})$ \\
\hline Baseline & $1.0(1.8,0$ to 13.7$)$ \\
5 years & $2.8(5.3,0$ to 36.6$)$ \\
10 years & $5.8(10.0,0.6$ to 46.1$)$ \\
14 years & $9.4(12.5,1.0$ to 46.8$)$ \\
Change from baseline to 5 years & $1.6(3.6,-0.4$ to 22.9$)$ \\
Change from 5 to 10 years & $2.7(4.6,-0.3$ to 29.3$)$ \\
Change from 10 to 14 years & $1.5(2.6,-4.1$ to 12.9$)$ \\
\hline
\end{tabular}

Values are median (mean, range).

14 year follow up. With estimated atrophy as the outcome, the baseline values and the three approximately five year changes in log transformed lesion loads were included as predictors. Log transformation improved the normality of the regression model residuals, adding greater confidence to the model fit, and reduced dependence on extreme values. Where the lesion volume was zero, the log volume was imputed by randomly drawing from a normal distribution, with mean and standard deviations estimated from a tobit model, ${ }^{14}$ left censored at the lowest non-zero log lesion load value, randomly selecting values drawn below this lower limit. This yielded conservative estimates of the regression slopes.

\section{RESULTS}

In normal control subjects, the estimated mean difference in BPF between male and female subjects was 0.009 (about 1\%), and age effects were similar in both sexes (both -0.002 per year, or around $-0.3 \%$ per year). These effects were allowed for when assessing changes in BPF associated with multiple sclerosis.

On average, T2 lesion loads increased in each of the five year intervals studied, although there was quite marked intersubject variability in the magnitude of these changes (table 1). BPF was on average lower in the multiple sclerosis group (mean 0.79 , median 0.80 , range 0.70 to 0.85 ) than in the control group (mean 0.84, median 0.85, range 0.78 to 0.87 ) and, allowing for age and sex, the differences were estimated to be -0.03 (or around $-3.6 \%, p=0.001$ ).

Linear regression modelling indicated that for estimated disease related reductions in BPF, the baseline and 0 to 5 year changes in log transformed lesion load were significant predictors, with the 5 to 10 year change being of borderline significance (baseline coefficient $(\beta)=-0.0203$ (95\% confidence interval (CI) -0.0314 to -0.0092$), \mathrm{p}=0.001 ; 0$ to 5 year $\beta=-0.0284(-0.0450$ to -0.0119$), p=0.002$; and 5 to 10 year $\beta=-0.0143(-0.0302$ to +0.0015$), p=0.074$; overall model $\left.R^{2}=0.4363, \mathrm{p}=0.003\right)$. The significance of the contribution of the 5 to 10 year changes in lesion loads towards the prediction of BPF was heavily dependent on a single outlier and should be regarded with caution. These results reflect patterns observed in the Spearman correlations (table 2, fig 1). Zeros were imputed for four subjects at baseline and one subject at five years, because they had no lesions on the corresponding scan. No subjects required imputation of zeros at 10 or 14 years.

\section{DISCUSSION}

With longer follow up, there appears to be an increasingly strong correlation of separate time point T2 lesion volume measures with estimated disease related brain atrophy at 14 year follow up, the main increase being apparent between baseline and five years (table 2, fig 1). Further, there appeared to be a declining relation between change in $\mathrm{T} 2$ lesion load and estimated disease related atrophy at the 14 year follow up. Considered together with the results of 
Table 2 Spearman correlations between T2 lesion loads at various time points and estimated atrophy of brain parenchymal fraction at the 14 year follow up

\begin{tabular}{ll}
\hline T2 lesion load & Spearman correlation \\
\hline Baseline & $-0.262(-0.586$ to +0.135$), 0.178$ \\
5 years & $-0.497(-0.739$ to -0.141$), 0.007^{*}$ \\
10 years & $-0.506(-0.745$ to -0.153$), 0.006^{*}$ \\
14 years & $-0.530(-0.759$ to -0.184$), 0.004^{*}$ \\
Change from baseline to 5 years $-0.528(-0.758$ to -0.182$), 0.004^{*}$ \\
Change from 5 to 10 years & $-0.326(-0.630$ to +0.065$), 0.090$ \\
Change from 10 to 14 years & $-0.016(-0.397$ to +0.369$), 0.936$ \\
\hline
\end{tabular}

Values are: mean (95\% confidence interval), two tailed $p$ value. ${ }^{*} \mathrm{p}<0.05$.

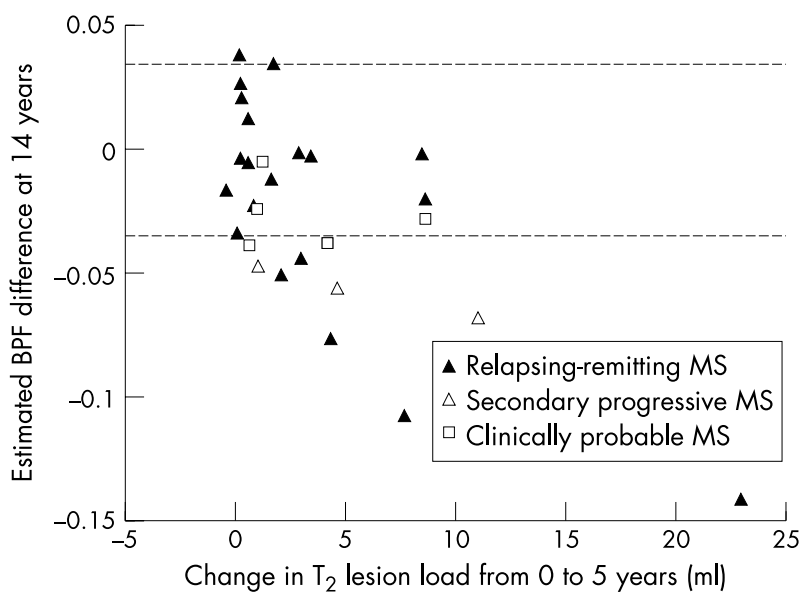

Figure 1 Estimated disease related effects upon brain parenchymal fraction (BPF) at 14 year follow up (follow up subjects compared with projected normal control values for a given subject's age and sex) against change in T2 lesion load between baseline and five years. The dashed horizontal lines represent the limits within which $95 \%$ of differences between projected BPF (allowing for age and sex) and measured BPF in control subjects should fall. Lesion load data were log transformed before assessing associations with fractional tissue volumes.

regression modelling, this suggests that in this cohort early rather than later focal lesion accumulation was related to the subsequent course of brain atrophy. This result reflects the observations from a larger cohort showing that early change in lesion load was more strongly related to disability at 14 years than later change in lesion load. ${ }^{9}$ With these data, it is not possible to ascertain when this atrophy begins, although previous work on cohorts with both clinically isolated syndrome ${ }^{15}{ }^{16}$ and early multiple sclerosis ${ }^{12}{ }^{17}$ has shown that atrophy may be present very early in the clinical course of the disease.

When considering pathological explanations for relation between focal lesions and brain atrophy it should be recalled that, given the present evidence, neither measure may be pathologically specific. T2 lesions appear to be quite heterogeneous, with contributions from inflammation, oedema, demyelination, and axonal loss. ${ }^{18}$ Brain atrophy, while also being influenced by similar factors, appears to be more specifically weighted towards neurodegeneration. ${ }^{1}$

The relations between focal lesion genesis and brain atrophy may be both direct and indirect. Acute inflammatory lesions are associated with axonal dysfunction ${ }^{19}$ and transection, ${ }^{20}$ and this in turn may lead directly to anterograde and retrograde degeneration of axons with associated tissue volume loss. However, there may also be an indirect link, potentially with an associated delay. Demyelinated axons may not be lost immediately but may degenerate later. ${ }^{21}$ This could reflect loss of oligodendrocyte support ${ }^{22}$ or increased vulnerability to other stress factors such as nitric oxide. ${ }^{23}$

The regression model $R^{2}$ value (0.44) indicates that baseline and changing lesion loads explain less than $50 \%$ of variability in estimated disease related atrophy. This suggests that factors other than focal lesion accumulation play a potentially greater role in the determination of brain atrophy in multiple sclerosis. ${ }^{24}$ It also suggests that treatments could have differential effects on lesion genesis and atrophy, depending on their mechanisms of action.

When considering the results, there are several limitations that should be recalled. Scanner hardware, scan acquisition variables, and scan assessors changed over the follow up period, and so lesion volume measurement errors may differ in two ways. First, the relation between the observed and true T2 lesion load - that is, the bias of the measurements-may differ. This could alter the apparent magnitude of lesion load change with time but should not alter the relative ranking of subjects with regard to changing lesion loads, and so ought not to affect the strength of any associations with changing lesion load. Second, the precision of the lesion load measurements may also differ, and it would seem reasonable to assume that increasing magnetic field strength ${ }^{25}$ and decreasing slice thickness ${ }^{26-28}$ will reduce this component of measurement error. Given this, it may be expected that earlier measurements in our study would be less precise than later ones, and this would tend to reduce rather than accentuate the strength of any association between early change in lesion loads and estimated atrophy at 14 years. For these reasons, we doubt that the changes in scanner hardware or acquisition sequence during the study have contributed to the correlations observed between lesion load and atrophy.

We made use of cross sectional data to assess disease related atrophy, and caution should be used when attempting to infer rates of change from this analysis. Further, while age and sex were allowed for when comparing the index and control groups, the control subjects were on average younger (three of the 28 subjects with multiple sclerosis were above the age range covered by the control cohort), and this may have influenced the apparent magnitude of disease effects. However, this should have little effect on the strength of the associations with lesion loads, as the relative ranking of the subjects in terms of raw fractional tissue volumes or estimated atrophy will be similar. There was no evidence of confounding by age and sex when these were added to the regression models alongside the changes in log transformed T2 lesion volumes, further suggesting that this adjustment adequately controlled for normal age and sex effects.

An important limitation of our study was that the cohort was a relatively small subgroup of those originally recruited and followed up for one year (109 subjects), and also represents only $53 \%(28 / 53)$ of all clinically definite and probable subjects with multiple sclerosis about whom clinical data were available at the 14 year follow up. ${ }^{9}$ The present subgroup is biased towards subjects with a relatively benign disease course and includes only three with multiple sclerosis who had developed secondary progressive disease at 14 years. Further long term studies are needed to confirm our results, and in particular to explore the temporal relations between lesion load and atrophy in subjects who develop progressive forms of multiple sclerosis.

\section{Conclusions}

The results of our study suggest that atrophy in multiple sclerosis is partly linked to early rather than later focal lesion accumulation. The strength of these relations is modest, indicating that factors other than those leading to overt 
lesion formation may play a more significant role in determining the degree of brain atrophy in multiple sclerosis.

\section{ACKNOWLEDGEMENTS}

We thank the subjects who kindly agreed to take part in this study; the Multiple Sclerosis Society of Great Britain and Northern Ireland for programme grant support to the NMR Research Unit; Schering AG for their sponsorship of DC and PB; Teva Pharmaceutical Industries for their sponsorship of OC; and Elan Pharma for their sponsorship of CD.

\section{Authors' affiliations}

D T Chard, P A Brex, O Ciccarelli, C M Griffin, C Dalton, D R Altmann,

A J Thompson, D H Miller, NMR Research Unit, Institute of Neurology, University College London, Queen Square, London WC1, UK

G J M Parker, Imaging Science and Biomedical Engineering, University of Manchester, Oxford Road, Manchester, UK

D R Altmann, Medical Statistics Unit, London School of Hygiene and Tropical Medicine, Keppel Street, London WCl, UK

Competing interests: none declared

\section{REFERENCES}

1 Miller DH, Barkhof F, Frank JA, et al. Measurement of atrophy in multiple sclerosis: pathological basis, methodological aspects and clinical relevance. Brain 2002; 125:1676-95.

2 Kurtzke JF. Rating neurologic impairment in multiple sclerosis: an expanded disability status scale (EDSS). Neurology 1983;33:1444-52.

3 Poser CM, Paty DW, Scheinberg L, et al. New diagnostic criteria for multiple sclerosis: guidelines for research protocols. Ann Neurol 1983;13:227-31.

4 Miller DH, Ormerod IE, McDonald WI, et al. The early risk of multiple sclerosis after optic neuritis. J Neurol Neurosurg Psychiatry 1988;51:1569-71.

5 Miller DH, Ormerod IE, Rudge $\mathrm{P}$, et al. The early risk of multiple sclerosis following isolated acute syndromes of the brainstem and spinal cord. Ann Neurol 1989:26:635-9.

6 Morrissey SP, Miller DH, Kendall BE, et al. The significance of brain magnetic resonance imaging abnormalities at presentation with clinically isolated syndromes suggestive of multiple sclerosis. A 5-year follow-up study. Brain 1993:116:135-46.

7 Filippi M, Horsfield MA, Morrissey SP, et al. Quantitative brain MRI lesion load predicts the course of clinically isolated syndromes suggestive of multiple sclerosis. Neurology 1994;44:635-41.

8 O'Riordan JI, Thompson AJ, Kingsley DP, et al. The prognostic value of brain MRI in clinically isolated syndromes of the CNS. A 10-year follow-up. Brain 1998; 121:495-503.

9 Brex PA, Ciccarelli O, O'Riordan Jl, et al. A longitudinal study of abnormalities on MRI and disability from multiple sclerosis. N Engl J Med 2002;346:158-64.
10 Sailer M, O'Riordan Jl, Thompson AJ, et al. Quantitative MRI in patients with clinically isolated syndromes suggestive of demyelination. Neurology 1999;52:599-606.

11 Plummer DL. Dispimage: a display and analysis tool for medical images. Rev Neuroradiol 1992;5:489-95.

12 Chard DT, Griffin CM, Parker GJ, et al. Brain atrophy in clinically early relapsing-remitting multiple sclerosis. Brain 2002; 125:327-37.

13 Zar JH. Simple linear correlation. In: Zar JH, ed. Biostatistical analysis. New Jersey: Prentice-Hall, 1999:377-412.

14 Tobin J. Estimation of relationships for limited dependent variables. Econometrica 1958;26:24-6.

15 Brex PA, Jenkins R, Fox NC, et al. Detection of ventricular enlargement in patients at the earliest clinical stage of MS. Neurology 2000;54:1689-91

16 Dalton CM, Brex PA, Jenkins R, et al. Progressive ventricular enlargement in patients with clinically isolated syndromes is associated with the early development of multiple sclerosis. J Neurol Neurosurg Psychiatry 2002;73:141-7.

17 Zivadinov R, Sepcic J, Nasuelli D, et al. A longitudinal study of brain atrophy and cognitive disturbances in the early phase of relapsing-remitting multiple sclerosis. J Neurol Neurosurg Psychiatry 2001;70:773-80.

18 Barkhof $F$, van Walderveen $M$. Characterization of tissue damage in multiple sclerosis by nuclear magnetic resonance. Philos Trans $R$ Soc Lond B Biol Sci 1999;354:1675-86.

19 Ferguson B, Matyszak MK, Esiri MM, et al. Axonal damage in acute multiple sclerosis lesions. Brain 1997; 120:393-9.

20 Trapp BD, Peterson J, Ransohoff RM, et al. Axonal transection in the lesions of multiple sclerosis. N Engl J Med 1998;338:278-85.

21 Kornek B, Storch MK, Weissert R, et al. Multiple sclerosis and chronic autoimmune encephalomyelitis: a comparative quantitative study of axonal injury in active, inactive, and remyelinated lesions. Am J Pathol 2000:157:267-76

22 Yin X, Crawford TO, Griffin JW, et al. Myelin-associated glycoprotein is a myelin signal that modulates the caliber of myelinated axons. J Neurosci 1998; 18:1953-62.

23 Smith KJ, Kapoor R, Hall SM, et al. Electrically active axons degenerate when exposed to nitric oxide. Ann Neurol 2001;49:470-6.

24 Kalkers NF, Vrenken H, Uitdehaag BM, et al. Brain atrophy in multiple sclerosis: impact of lesions and of damage of whole brain tissue. Mult Scler 2002:8:410-14.

25 Filippi $M$, van Waesberghe $\mathrm{JH}$, Horsfield MA, et al. Interscanner variation in brain MRI lesion load measurements in MS: implications for clinical trials. Neurology 1997;49:371-7.

26 Filippi M, Rovaris M, Sormani MP, et al. Intraobserver and interobserver variability in measuring changes in lesion volume on serial brain MR images in multiple sclerosis. Am J Neuroradiol 1998;19:685-7.

27 Filippi M, Marciano N, Capra R, et al. The effect of imprecise repositioning on lesion volume measurements in patients with multiple sclerosis. Neurology 1997:49:274-6.

28 Molyneux PD, Tubridy N, Parker GJ, et al. The effect of section thickness on MR lesion detection and quantification in multiple sclerosis. Am J Neuroradiol 1998;19:1715-20. 